\title{
UN ESTUDIO EXPLORATORIO SOBRE LA RELACIÓN ENTRE LA LEGITIMIDAD INSTITUCIONAL Y LA TRANSGRESIÓN NORMATIVA EN ARGENTINA
}

\author{
AN EXPLORATORY STUDY OF THE RELATIONSHIP BETWEEN INSTITUTIONAL \\ LEGITIMACY AND NORMATIVE TRANSGRESSION IN ARGENTINA
}

\author{
Maite Regina Beramendi \\ Elena Mercedes Zubieta \\ Universidad de Buenos Aires (UBA) \\ Consejo Nacional de Investigaciones Científicas y Técnicas (CONICET), Argentina
}

\begin{abstract}
Resumen: El presente trabajo busca conocer cómo un grupo de estudiantes universitarios caracteriza el sistema normativo en Argentina. Para tal fin, se analizaron las percepciones acerca de la transgresión normativa, la legitimidad del sistema y la vinculación de ambos aspectos. Los resultados muestran que los participantes desconfían del funcionamiento del sistema normativo, pues éste se maneja mediante prácticas corruptas, a través de instituciones ineficaces y con un sistema de dobles normas, piensan, a su vez, que el sistema está pensado para la conveniencia de los políticos y de los ciudadanos con poder. Básicamente, creen que el sistema es injusto e ilegítimo. Los estudiantes dan respuestas cínicas en relación a un posible cambio, piensan que la forma de conducirse en el sistema es mediante una moralidad individual, con un sistema propio de normas que contempla la transgresión y que, al mismo tiempo, procura no perjudicar a alguien en concreto.
\end{abstract}

Palabras Clave: instituciones; legitimidad; norma perversa; transgresión normativa

\begin{abstract}
The present study investigates how a group of college students characterizes the normative system in Argentina. To this end, the perceptions of normative transgression, the legitimacy of the system and the entailment of both aspects were analyzed. The results show that participants distrust of the normative system's running as it is handled by corrupt practices, ineffective institutions, and a system of double standards. They also express that the system is thought for the convenience of the politicians and citizens with power. Basically, participants do not perceive the system as just or legitimate. Also, the students give cynical responses in relation to a possible change, holding the belief on an individual morality to cope with the system with particular norms that contemplate the transgression, if no one in concrete is harmed.
\end{abstract}

Keywords: institutions; legitimacy; normative transgression; perverse norms

La sociedad está organizada por un Estado, que provee una estructura social y un marco de legalidad para que condiciona las dinámicas sociales (Nino, 2005). Desde la perspectiva sociológica, Durkheim (1897/1998) analiza cómo la sociedad condiciona a los individuos. Según el autor, la sociedad está conformada por un conjunto de sentimientos, ideas, creencias y valores que organizan y determinan los comportamientos de las personas. Además, el autor especifica que las funciones de la sociedad son integrar y regular a sus miembros, y plantea que cuando esta tarea no se desarrolla eficazmente los individuos perciben una situación de anomia. Por otro lado, Merton (1964, como se citó en Wolfe, 1968) plantea que la anomia es el producto una discrepancia o conflicto entre las metas social- mente determinadas y el sentido socialmente establecido.

En el área de la psicología social, Srole (1956) tomó el concepto de anomia formulado por Durkheim (1897), para centrarse en indagar cómo las personas evalúan subjetivamente el funcionamiento de la sociedad y cómo esto repercute psicosocialmente. Según el autor, la anomia hace que las personas perciban un entorno desintegrado y una falta de implicación social, y repercute en una integración negativa de los individuos en la sociedad. Desde esta perspectiva se desarrollaron diversos trabajos que profundizan en el análisis de la anomia como noción psicosocial enfatizando el estudio de los sentimientos y percepciones individuales, que dan cuenta de la falta de apego al entorno, falta

Correspondencia: Maite Beramendi. Universidad de Buenos Aires - CONICET.

Correo Electrónico: maiteberamendi@gmail.com 
de involucramiento, soledad, entre otras emociones (Abello et al., 2010; Aceituno et al., 2010; Beramendi, Sosa \& Zubieta, 2012; Blank, 2003; Laca Arocena, Mejía Ceballos \& Yañez Velasco, 2010; Muratori, Delfino \& Zubieta, 2013).

Los estudios en el contexto argentino dan cuenta de altos niveles de percepción de anomia, que indican creencias sobre disfuncionalidad institucional y una integración deficitaria en el sistema social (Beramendi et al., 2012), así como un déficit en lo que hace a la aceptación social, al logro de confianza y actitudes positivas hacia los otros y una baja confianza en las instituciones (Muratori et al., 2013).

Sin embargo, en la década del '80 del siglo pasado en el área de las ciencias políticas, el concepto de anomia comenzó a debilitarse, ya que el bajo cumplimiento no se asociaba con la ausencia de normas. Como explica O’Donnell (1996) en lo que respecta a la cultura institucional argentina, en tanto norma situada, no es que no existan normas reguladas por instituciones formales sino que, por el contrario, se crean numerosas normas y leyes que buscan reglamentar el comportamiento social pero que no son respetadas por los ciudadanos.

Lauth (2000) plantea que el incumplimiento normativo no puede explicarse a través de la ausencia de normas ya que las personas responden a patrones de comportamientos ordenados, sino que el problema radica en que las personas siguen y cumplen las normas que provienen de instituciones informales. Estas son reglas socialmente compartidas, generalmente no escritas y son aplicadas fuera de los canales sancionados oficialmente (Helmke \& Levitsky, 2004).

El poder de las instituciones informales deviene generalmente, porque las instituciones formales son ineficientes y/o ineficaces. El problema radica en que las instituciones informales conviven con las formales pero no las reemplazan, generándose así dobles normativas (Helmke \& Levitsky, 2004).

Dentro del marco de la psicología social también se analiza esta doble normatividad. Fernández Dols $(1992,1993)$ propone analizar el comportamiento transgresor no como un problema individual sino como la resultante de dinámicas sociales perversas. Según el autor, en las sociedades se crea una norma perversa cuando una norma explícita es incumplible porque un grupo asume o sufre la presión de otro grupo. Como estas normas no pueden cumplirse se crean normas alternativas que son aquellas que las personas reconocen. La perversidad radica en que las normas explícitas no se anulan sino que conviven con las alternativas, superponiéndose y sin saber si en algún momento no se recibirá una sanción por ello. Al mismo tiempo, diversos grupos pujan por controlar las normas alternativas $y$, de esta manera, nacen asociaciones como el amiguismo, el clientelismo o el nepotismo, entre otras.

Desde la Teoría Focal de la Conducta Normativa (Cialdini, Reno \& Kallegren ,1990), Cialdini et al. (1990) explican que las normas sociales no son conceptualizadas meramente como aquello que se debería hacer sino que se catalogan de dos maneras: (a) las normas prescriptivas, que refieren a la percepción de uno sobre lo que el resto considera una conducta apropiada; y (b) las normas descriptivas, que refieren a lo que la mayoría realmente hace. A pesar de que ambas normas se relacionan, suponen conceptualizaciones y motivaciones independientes. Las normas sociales prescriptivas guían la conducta de las personas a través de una evaluación social. En cambio, las normas sociales descriptivas conducen a las personas a actuar a través de la información social que permite comprender aquella conducta que es adaptativa y eficaz al contexto (Cialdini, 2007).

En este sentido, las normas informales que las personas respetan podrían ser conceptualizadas como normas sociales descriptivas que enmarcan el funcionamiento normativo social (Beramendi \& Zubieta, 2015). Sin embargo, White, Smith, Terry, Greenslade y McKimmie (2009) plantean que hay un tipo de norma que no responde a variables contextuales y las denominan normas prescriptivas personales o morales. Estas son reglas internalizadas por el individuo que sirven como parámetro para aprobar o desaprobar la realización de la propia conducta, más allá de las repercusiones personales o sociales (White et al., 2009). Estas normas explicarían en gran medida por qué la gente cumple o incumple ciertas normas (MacCoun, 1993), más allá de la situación específica, y sobre todo, excediendo las evaluaciones de costo y beneficio (Manstead, 2000). Según Sacconi y Faillo (2010) la motivación intrínseca para cumplir las normas es importante, sobre todo, cuando en la sociedad la transgresión normativa es alta.

Sin embargo, en el contexto argentino se considera que la transgresión es una práctica común y arraigada. En el país se visualizan cotidianamente todo tipo de transgresiones 
que van desde el incumplimiento de normas de convivencia hasta actos de corrupción política. Esto ha permitido hablar de una cultura de la transgresión argentina (e.g., De Biase, 2010; Latinobarómetro, 1996-2010; Nino, 2004) y hasta identificar la transgresión como un rasgo idiosincrático del argentino que cobra vida a través de la denominación el argentino "avivado" (Aguinis, 2001; Denevi, 2002; Jauretche, 1973; Mafud, 1965; Monsegur et al., 2014; Roselli, 2000). Por lo tanto, la transgresión normativa en la Argentina no puede ser pensada desde una perspectiva individual sino como una respuesta colectiva a una dinámica institucional que posee dobles normas.

El poder de las instituciones informales trae diversas consecuencias negativas, sobre todo cuando su uso es generalizado (Helmke \& Levitsky, 2004). En principio, las instituciones formales pierden un poder real ya que existen pero no son respetadas. En segunda instancia, las instituciones comienzan a estructurarse y regularse por canales que no están controlados por las leyes que deben proteger el funcionamiento social y esto conlleva a prácticas de corrupción, clientelismo o nepotismo (Helmke \& Levitsky, 2004). Por ejemplo, en las universidades se deben realizar concursos que avalen las elecciones de los profesores pero si estos concursos no se llevan a cabo, los contratos de los profesores se eligen a partir del interés de un directivo o profesor catedrático. Este tipo de regulación puede conducir a prácticas como el amiguismo o 'clientelistas', sobre todo, ya que lo que se pierde es el control institucional. En estas circunstancias pasan a tener más poder quienes controla las instituciones que las normas institucionales en sí mismas.

Por otro lado, las instituciones y sus autoridades pierden la legitimidad que deberían poseer y esto acrecienta la probabilidad de que se transgredan las normas (Tyler, 2001, 2006). Cuando las personas perciben que las instituciones y sus autoridades son justas, tienden a cumplir en mayor medida las normas; sin embargo, cuando conciben que son ilegítimas, hay mayores posibilidades de que la gente transgreda las normas. Según Napier y Tyler (2008) el efecto de la percepción de legitimidad genera que las personas se sientan satisfechas con los procesos de toma de decisiones y acepten voluntariamente los resultados que se producen a partir de las instituciones. Sin embargo, cuando las personas perciben mecanismos de corrupción en el
Gobierno, baja eficacia y confianza institucional, procesos de justicia distributiva y procedimental incorrectos y autoridades autoritarias, la gente tiende a transgredir en mayor medida las normas (c.f., Baker, 2008; Brandt, 2013; González de la Vega, Quintanilla \& Tajonar, 2010; Mueller \& Landsman, 2004; Murphy \& Tyler, 2008; Napier \& Tyler, 2008; Rose-Ackerman, 2001; Tyler, 2001, 2006). En otras palabras, la legitimidad institucional y de las autoridades genera que las personas cumplan en mayor medida las normas.

Cuando se ve comprometida la legitimidad de las instituciones de una sociedad, se pone en discusión la justificación de su estatus quo. Es decir, la gente va a buscar el cambio social a través de la transgresión (Wright, Taylor \& Moghaddam, 1990). Para Wright (2001) cuando las personas perciben que es legítimo el funcionamiento de la sociedad, las personas realizan acciones colectivas o individuales aceptadas socialmente para cambiar las condiciones de cierto grupo social o el propio; sin embargo, el autor plantea que cuando la gente descree del funcionamiento del orden social, esta realiza acciones que buscan el cambio pero violando ciertas normas sociales de manera individual o colectivamente.

Según Wright y Taylor (1998), el problema radica en que si las personas eligen realizar acciones no normativas individuales se perpetúa el funcionamiento de la sociedad y no se discute colectivamente aquellos cambios necesarios para mejorar institucionalmente los problemas que acarrea la sociedad. Es decir, no se posibilita el cambio social.

En el contexto argentino, Alonso y Brussino (2012) analizaron la legitimidad de las autoridades legales de la provincia de Córdoba (e.g., policía y funcionarios) y del sistema de justicia procedimental. Los resultados indicaron que los participantes manifiestan una valoración negativa de las autoridades y del sistema de justicia, que darían cuenta de índices bajos de percepción de legitimidad.

Por otro lado, en el contexto local también se percibe altos niveles de corrupción (e.g., COPUB, 2013), baja confianza institucional, sobre todo en los poderes centrales del Estado (e.g., Edelman, 2013; Observatorio de la Deuda Social Argentina, 2012). Sin embargo, según estudios locales los jóvenes universitarios perciben menores niveles de corrupción, falta de legitimidad institucional y de las autoridades y menor grado de confianza institucional que la población de mayor edad con 
y sin estudios universitarios (Beramendi, 2014).

En este marco, se realizó un estudio con el objetivo de indagar cómo caracterizan al sistema normativo argentino un grupo de jóvenes universitarios. Se buscó comprender qué entendían por norma, cómo describían el funcionamiento del sistema institucional y si percibían este sistema como legítimo.

\section{Método}

\section{Contexto}

Los sujetos fueron contactados personalmente, por invitación de un profesor o un compañero que les recomendó la actividad, y su participación fue voluntaria.

Las actividades se realizaron en las universidades a las cuales pertenecían los alumnos: (a) Instituto de Investigaciones de Psicología de la Universidad de Buenos Aires (UBA); (b) Instituto de Investigaciones de Psicología y Educación de la Universidad Católica Argentina (UCA). Las actividades tuvieron una duración entre 60 y 70 minutos.

\section{Participantes}

La muestra fue intencional, compuesta por 30 alumnos universitarios (13 hombres y 17 mujeres), entre 20 y 28 años, de los cuales 15 pertenecían a la Universidad de Buenos Aires y 15 a la Pontificia Universidad Católica Argentina. Los participantes cursaban la Carrera de Psicología, excepto una que pertenecía a la Carrera de Letras (UBA).

\section{Diseño}

Para el análisis de la información obtenida en los grupos de discusión se contempló el modelo de diseño denominado emergente-sistemático de grupos focales, lo emergente refiere a los grupos que se usan con fines exploratorios, mientras que sistemático refiere a los grupos que se utilizan con propósitos de verificación (Onwuegbuzie, Dickinson, Leech \& Zoran, 2011). Este diseño se complementa con el sustento teórico de la Teoría Fundamentada, en el cual hay una comparación y análisis constante de las categorías que se crean (Onwuegbuzie et al, 2011). Este método utilizado para el análisis de investigaciones cualitativas, permite desarrollar una teoría a partir de los datos recopilados de manera sistemática y analizados por medio de un proceso de investigación (Strauss \& Corbin, 2002).

\section{Técnicas y herramientas de recolección de información}

Para la recolección de información se utilizó la técnica de grupo de discusión, que consiste en reuniones grupales en las cuales los participantes conversan sobre temas sugeridos por el coordinador del grupo (Onwuegbuzie et al., 2011). Se elaboró una guía de entrevista que contemplaba preguntas sobre los siguientes temas: (a) concepciones de la norma (Definición de la norma), (b) representaciones del cumplimiento normativo (Respeto y Transgresión), (c) percepción del sistema normativo (Confianza institucional y Doble Funcionamiento Normativo), (d) emociones que emergen de la dinámica del Sistema Normativo (Sensación de Desmoralización) y (e) posibilidad de Cambio (Cambio Social).

\section{Procedimiento}

Las actividades se realizaron en las universidades a las cuales pertenecían los estudiantes. Específicamente se realizaron 4 sesiones de discusión grupal que tuvieron una duración aproximada de 65 minutos.

Las sesiones de discusión grupal fueron grabadas y contaron a su vez con colaboradores que anotaron elementos considerados relevantes, incluyendo los datos socio-demográficos de los participantes. Se utilizó el análisis basado en la transcripción (Onwuegbuzie et al., 2011) que fue procesado mediante el programa Atlas $\mathrm{Ti}$, que es un programa computacional que posibilita segmentar los datos en unidades, codificar datos y construir teoría (Muñoz Justicia, 2005). Sin embargo, por el objetivo de este estudio y las características de la herramienta de recolección de datos, se focalizó en el análisis temático que surgió de la coincidencia y disidencia de los participantes, y no tanto en el análisis de las diferencias de la muestra.

\section{Resultados}

A partir del análisis de la información recabada en los grupos focales, se crearon 8 categorías que refieren específicamente a los objetivos aquí desarrollados: 1 . Concepto de norma; 2. Doble funcionamiento normativo; 3 . Trasgresión 
generalizada de las normas; 4. Falta de control y sanción en el incumplimiento de las normas; 5. Falta de confianza en el sistema; 6 . Sensación de desmoralización; 7. Respeto por las normas; y 8. Posibilidad de cambio social.

1. Concepto de norma: definición que las personas daban sobre la norma. Se dividió en dos sub-categorías:

a. Norma como pauta social: los participantes consideran a la norma como una pauta o criterio común que organiza socialmente a las personas. Se diferencia de una ley porque las normas pueden tener un carácter implícito, no escrito, y un castigo informal, como la exclusión social, y no meramente penal. Asimismo, reconocen que hay muchos estilos de normas: sociales, familiares, jurídicas, etc.

Ejemplos: (a) "convenciones de la sociedad para llegar a un fin común determinado"; y (b) "tendrían que ser las cláusulas del contrato que es la sociedad".

b. Norma en la Argentina: los participantes las consideran entes aislados y desagregados, sin una coherencia entre sí, carentes de una organización por parte del sistema normativo. Los estudiantes expresaban adjetivos como: ridículas, inventadas, negativas, para pocos, sin sentido y dependientes de un contexto cambiante, etc. Se las concibe como una imposición, un castigo o una limitación, y se la asocia a prácticas autoritarias.

Ejemplos: (a) "Sí, que había dicho que en Francia o en otro lugar las leyes de tránsito estaban ligadas al sistema de salud, entonces, como las normas en sí parecen ser un cuerpo coherente de leyes y normas que si cae una, las otras dejan de tener sentido. Y acá parecería que las normas son como entes aislados y desagregados entre sí que no tienen vinculación con nada, que si sube una, baja otra, que si cumplo una, no cumplo otra, no importa porque no hace a la entereza de un cuerpo social, y eso hace a lo de individualistas y colectivistas también en las mismas normas"; y (b) "por ahí sí, nosotros vamos adaptando las normas pero en realidad no debería ser así porque sino no existe la norma sino que vos vas teniendo tus parámetros de norma y entonces da lugar a la norma, de la norma, de la norma, y que se yo, que funciona así es una cosa, que se da mucho en Argentina, que la norma deja de ser la norma y la norma es la que nos parece. $Y$ después, me parece que es lo que debería ser y que me parece que se ve en otros países, y es que si tenés que cruzar en una esquina a nadie se le ocurre cruzar por la mitad de la calle. La gente en general por ahí respeta más, por ahí te dicen que 'no toque la bocina', y acá nos conviene tocar la bocina en ese momento y la tocamos".

2. Doble funcionamiento normativo: Se desglosa en dos subcategorías:

a. Doble norma: los estudiantes creen que las instituciones argentinas tienen un doble funcionamiento: (a) el formal, que normativiza cómo deberían funcionar las instituciones; y (b) el informal, que organiza el funcionamiento real de las instituciones. Ambas normativas se contraponen y superponen, generando una situación disruptiva pues las personas deben manejarse según las normas informales pero no saben si serán sancionadas por ello, ya que las normas en vigencia son las formales.

Ejemplos: (a) "(...) doble norma, que me parece también interesante, el hecho de que hay una norma pero después se hacen otras cosas, pero esas cosas que después se hacen de esa manera ¿me explico? Hay una ley que dice que las cosas se hacen así y hay un submundo en el cual la institución; por ejemplo, el estatuto universitario dice que las cosas se resuelven de determinada manera, después los docentes se manejan de otra, y hay un gremio y un montón de cosas que van haciendo que uno tenga que aprender el doble funcionamiento: la norma que está y la norma que se cumple"; y (b) "yo creo que sí, como que el sistema está, no sé, como que está preparado para que la gente transgreda, lo veo mucho en las 'coimas' por ejemplo, si querés sacar el documento: hacés una fila de 5 cuadras o le pagas a un comisario una plata y te lo dan en dos días y no hacés la cola; y como que está preparado así muchas cosas (...) pero por ejemplo en un país del primer mundo no te van a hacer hacer una cola de 5 cuadras. Hay cosas que no te facilitan, si te pondrían otra oficina más donde atender a más personas en menos tiempo. Como que también uno cuando paga impuestos, para que pongan otra oficina y que lo atiendan mejor a uno".

b. Emociones que genera el doble funcionamiento: los participantes comentaron que el doble funcionamiento normativo genera emociones negativas como enojo, ansie- 
dad, acostumbramiento, impotencia, etc. Ejemplos: (a) "Algo de ansiedad, es el clásico. Pensando desde un jefe, un jefe que tiene normas claras podés estar a gusto o a disgusto, podés cuestionarlas, pero las normas son claras. Después cuando las normas no son claras, se genera más ansiedad porque uno no puede prever, no puede hacer un pronóstico de cuál es el diagnóstico, no puede hacer un pronóstico de qué va a pasar si yo hago tal o cual cosa. Ansiedad"; (b) "creo que en algunos casos enojo, enojo, $y$ al final acostumbramiento. Yo tengo a mi hermana que es profesora de inglés que da clases en la secundaria y supuestamente los chicos, supuestamente tiene que aprobar con 7 , como cualquier secundario, y le bajaron la orden del gobierno, de la secretaría de educación de que ella no podía reprobar a ningún chico, o sea que el chico sepa o no sepa, tiene que aprobar igual, y es un secundario público. $Y$ te digo, al principio mi hermana mucho enojo, 'putea', pero al final tiene que terminar porque sí, baje de donde baje y es así. Y termina adaptándose al sistema hasta el punto, no tanto porque ya no quiere enseñar en secundario, porque ella lo vive como un fracaso personal, muy frustrada. Creo que en algunos se vive como frustración y otros, como amigos de ella, se adaptan, y los chicos salen recibidos, con el título secundario sin saber ni siquiera, con errores de ortografía, sin saber inglés, pero sí".

\section{Trasgresión generalizada de las normas:} incumplimiento constante y generalizado de la norma. Esta categoría se subdivide en tres subcategorías:

a. Incumplimiento generalizado: los estudiantes perciben que las normas son constantemente vulneradas y de manera generalizada hasta tal punto que los participantes reconocen que todas las normas pueden ser trasgredidas.

Ejemplos: (a) "Estaba hablando yo, de que lo normal es no seguir la norma pero siempre teniendo en cuenta que hay una norma, no es anarquía, la norma está pero lo gustoso es correrse, lo gustoso o lo interesante o lo 'piola' de lo prohibido (...) Pero hay algo de eso, la norma tiene que estar para que se infrinja"; (b) "Entrevistados: ¿Se imaginan, ya para ir cerrando, que haya normas que se cumplan sí o sí? Participantes: no (general), Participante: como que cualquier cosa se puede trasgredir". b. Justificaciones del incumplimiento: a la hora de exponer las causas del incumplimiento los alumnos aludieron a ausencia de premio y castigo en relación con el cumplimiento e incumplimiento, carencia de retribuciones a los aportes, falta de coherencia de las normas, corrupción, incumplimiento de la norma por la autoridad, escepticismo con respecto al sistema, y la percepción de un sistema legal injusto.

Ejemplos: (a) "para mí tiene que ver también por las formas de funcionamiento de este país. Donde se estableció, que se yo, como tantos niveles de corrupción que la gente piensa, que bueno que si no lo hago yo lo hace otro "; y (b) "me parece que es importante, yo sigo con la confianza institucional, porque la norma, porque hablábamos de autoridad, de representatividady de que haya consenso; y si, cuando, no digo que sea el motivo por el cual trasgredimos todas las normas, pero cuando se ve ahí, que se ve mucho, que se trasgreden las normas, es más difícil, no digo que sea el único motivo, para incumplir la norma. Creo que hay más motivos, cuestiones culturales y por cuestiones que hablábamos antes que seguramente influyen mucho también".

c. Venganza indirecta - "quid pro quo": los participantes manifestaron que perciben que mediante los mecanismos formales no se pueden generar cambios. Por lo cual, cuando creen que están siendo perjudicados por el sistema lo atacan de manera indirecta e individual con una acción que trasgrede una norma, que no resuelve el problema que los aqueja pero perjudica al sistema para sentirse resarcidos por lo que sienten que se les negó.

Ejemplos: (a) "Imaginate como persona que trabajás todos los días de la semana, que pagás tus impuestos, que estás todo al día, y así y todo, tenés que estar pendiente de tu seguridad porque podés salir a la calle y te pueden matar, porque te pueden robar, entonces tenés una impotencia frente a todo esto. Entonces empezás a no cumplir las normas si a vos no te están dando lo que vos necesitás, entonces empezás a reevaluar un montón de cosas, como no pagar los impuestos"; y (b) "claro, la 'avivada' porteña, de los argentinos. También en el trabajo 'me imprimo todo el material en las horas de trabajo، que se yo, también es irse de las normas. Llamo por teléfono porque total como a mí me explotan, los 'cago" llamándoles por teléfono. Se juegan esas cosas". 
4. Falta de control y sanción en el incumplimiento de las normas: los estudiantes universitarios explicitaron la falta de control y sanción generalizados de las normas. Se divide en tres sub-categorías:

a. Falta de sanción: los participantes piensan que el incumplimiento de las normas no es castigado y que su cumplimiento no es valorado, tanto a nivel institucional como social, en casi todos los ámbitos de la sociedad.

Ejemplos: (a) "Yo estaba pensando lo que se ve en la tele, digamos con el tema de las normas no pasa nada, los 'chorros' entran y salen de la calle, hay un montón de cosas que van haciendo un círculo vicioso. Y nadie va a pagar por la norma que infringe, entonces por qué no infringirla, es como que se crea un conflicto de intereses"; (b) "yo creo que las normas nos son castigadas en general, como que las podés manejar, y eso hace que haya mucha impunidad, digamos tampoco no es que no hay castigo para nada, pero es para pocos y se puede zafar".

b. Falta de control: los estudiantes creen que las personas pueden regirse por sus propios intereses ya que no hay entes que regulen las normas o leyes. En general, los participantes se sienten más controlados en ámbitos privados, como en empresas, o cuando el control lo ejerce el grupo. Es decir, cuando éste es más directo y personal. Ejemplos: (a) "En empresas o vas a un bar, algo que es de alguien y cumplís más porque tenés a alguien más directo que te vigile. Lo otro es como una gran cosa donde no hay un control directo, que queda muy en abstracto el control y si no te controlás vos es como que no te controla nadie y lo hago, qué sé yo, si no me ve nadie"; y (b) "incluso hasta policía no usa el cinturón, quien tendría que dar el ejemplo tampoco lo hace, entonces no se ¿a quién seguimos? Y quizás países como Suiza que son el ejemplo de la organización, viven de una manera recontra ordenada y ya no necesitan que los vigilen. Todos, y quizás las mismas normas, no se yo no las conozco, pero como que hay otra mentalidad si los tenemos que comparar".

c. No hay control entre ciudadanos: más allá de la falta de control institucional, los participantes perciben que hay una norma social por la cual uno no puede exigirle a quien infringe una norma que la cumpla. Tácitamente, esto implica que "uno no se mete en lo que hace el otro". Sienten que tienen derecho a reclamar cuando ven que están perjudicando a alguien vulnerable, como es el caso de fumar al lado de una embarazada. Ejemplos: (a) "Pero por ejemplo si yo estoy en un lugar que están fumando, y a mí me molesta, yo no fumo, creo que yo llegaría a decirle algo a esa persona si yo estoy con una mujer embarazada, yo creo que sí le diría 'mirá, ubicate, acá no fumés porque está la chica embarazada! Pero si a mí no me afecta directamente mal, mal, yo no le voy a decir nada porque no sé cómo va a reaccionar"; (b) "(...) a eso iba, te importa que el otro la cumpla pero vos tampoco la cumplís. Si vos no la cumplís, tenés que ser consciente que el otro tampoco la va a cumplir (...) por eso el hecho de pensar dos veces lo que hacés, en realidad lo ideal sería cumplir la norma como está establecida y no lo que sea más cómodo porque después va a venir una situación donde el otro no lo cumple y vos le tirás todos los bardos ${ }^{1}$ (i.e., te enojás con) al otro, pero vos en esa situación harías lo mismo".

5. Falta de confianza en el sistema: los estudiantes comentaron que hay una creencia generalizada de que el país no se rige por normas claras y eficaces, que hay prácticas corruptas que manejan el sistema, que las instituciones públicas son ineficaces y que el proceso judicial es injusto e inequitativo.

Ejemplos: (a) "Quería decir que la confianza en las instituciones es bastante baja y que eso también aporta a esta sensación de no consenso y transgresión de la norma"; y (b) "(...) es que pasa mucho con la incoherencia de las normas, las leyes, es que viene un gobierno y empieza algo, un proyecto social, obviamente en campaña política, lo deja por la mitad; cambia de gobierno y no se retoman esas cosas. Muchos técnicos o gente que está capacitada sobre un proyecto, sobre determinado estudio, y cambia de gobierno $y$ vienen los acomodados del nuevo gobierno $y$ los que estaban antes que 'por ahi' son súper capaces y que aprendieron un montón en todo el gobierno anterior, se van porque ponen a otros y empieza todo de cero. $Y$ así y creo que eso puede ser algo que influya en esto de falta de coherencia que se hace todo de a partes, no hay una dirección".

1. "Bardo", argentinismo, "balurdo", que significa problema, enredos. "Bardear" es quien genera problemas, enredos. 
6. Sensación de desmoralización: los participantes mencionaron emociones de desesperanza o desaliento que generan situaciones como la incertidumbre, el replanteo constante, la falta de coherencia del sistema, entre otras. Los participantes se sienten "tontos" si cumplen la norma, pues el nivel de incumplimiento y corrupción es tan alto que si ellos no infringen la norma, otro lo hará. Piensan que el sistema funciona así y que no pueden aportar soluciones para un cambio social.

Ejemplos: (a) "Sí, hay mucho de decir eso 'si al final yo soy un 'boludo' (tonto) que pago todo, que hago todo', qué sé yo, se escucha eso de que al final cumplir está mal porque la mayoría no lo cumple, es difícil, y para qué, no hay estímulo (...) también está eso de que podés llegar más arriba como esquivando un poco las normas. Podés saltearte un par de pasos y llegás más rápido"; y (b) "no sé, qué se yo, por ejemplo donde yo vivo en la esquina de mi casa hay una garita la pusimos con los vecinos (guardia de seguridad privada) y hace 2 semanas nos robaron el auto en la puerta de mi casa. Entonces no se, es como una cosa de desaliento de que, decir para qué, mientras que al final todos los que están con el de la Municipalidad de Lomas tienen autos nuevos, se hicieron la casa, la casita en la costa, hacen cruceros y lo muestran sin ningún problema y que se yo, es como que, sobre todo en mi familia trabajar y cumplir, pagar que se yo para qué, si al final ellos la hacen fácil y no cumplen las cosas que te piden a vos que cumplas. $Y$ eso genera mucho resentimiento en la gente y ganas de no cumplir nada porque ves que ellos no lo cumplen básicamente".

7. Respeto de la norma: variables que contemplan el cumplimiento de la norma. Se desglosa en dos subcategorías:

a. Criterio personal: los participantes manifestaron que cumplirían aquellas normas que concuerdan con los valores y creencias de las personas y se transgredirían aquellas con las cuales no acuerdan. También las personas trasgreden o no si creen que obtendrán un beneficio personal por ello. Ejemplos: (a) "si vos tenés un modelo a seguir, si el sistema sería bueno, creo que uno termina acomodándose. Tenés que tener un algo a seguir sino hacés lo que te conviene y es lo que estamos diciendo todos ¿qué hago? Y bueno, lo que me conviene a mí, sino me siento un 'boludo' si hago la fila, pero como tengo a mi tío que trabaja acá y bueno"; y (b) "yo me he encontrado a mi mismo justificando el incumplimiento de normas, de una manera muy elegante si se quiere, porque en última instancia me he encontrado haciendo lo que tenía ganas de hacer, y claro, realmente me descubrí a mi mismo en esa actitud, de decir, estoy justificando a expensas de autores geniales y de cualquier corriente, pero la verdad es que en última instancia también termino suscribiendo muchas veces a esta idea de que es posible construir tu propia norma, lo cual para vos solito. Es más, me he encontrado en más de una oportunidad, exigiendo normas que yo mismo no cumplo".

b. Depende del contexto y situación: los estudiantes dijeron que hay pocas normas que respetan constantemente, en casi todos los casos, a veces las cumplen y a veces no. En este sentido, el cumplimiento depende de variables contextuales, del análisis que realizan las personas en cada caso o de su estado emocional.

Ejemplos: (a) "yo pienso que sirven como regulación pero en un contexto particular. A mí me pasa con el semáforo, o sea, yo no quiero esperar, si veo que la calle está totalmente vacía la calle, no están cruzando autos, para mí tiene que ser relativa la norma. Entonces uno la trasgrede, pienso en la medida de las necesidades que tiene y cómo está adecuada la norma a la realidad digamos"; y (b) "es como que también las normas se rigen al lugar al cual se aplican. Porque la misma norma es en Conurbano, Banfield, Haedo, en Capital y en Puerto Madero se aplican totalmente distintas. Y creo que los argentinos tenemos la capacidad de cambiar que no tiene nadie, en Capital nos portamos de cierta forma, en Haedo de otra y en Puerto Madero de otra. Es como que tenemos una variabilidad, están las mismas en todos los lugares".

8. Posibilidad de cambio social: posibilidades o imposibilidades del cambio del funcionamiento normativo. Se divide en cuatro subcategorías:

a. Sensación de círculo vicioso: los participantes creen que hay un incumplimiento generalizado y no se vislumbra un cambio posible. Algunos apelan al propio comportamiento para generar un cambio haciendo hincapié en el ejemplo personal, aunque ven las limitaciones del caso único; mientras que otros claramente plantean que la acción de un individuo no genera un cambio por- 
que se estaría luchando contra todo un sistema abstracto, casi imperceptible que manipula las acciones de las personas. No vislumbran cómo generar un cambio y se encuentran ante una 'encerrona'.

Ejemplos: (a) "Creo que vale la pena cumplir la norma si nos ponemos de acuerdo en hacerlo todos, si lo voy a hacer yo sola no. Porque sé que si lo hago yo sola no voy a cambiar nada, no cambio nada porque el sistema sigue igual, y yo me perjudico, porque otros se benefician incumpliendo normas que yo cumplo"; (b) "(...) porque vos tratás de cambiar algo y la otra persona te está rompiendo las ilusiones y en el mismo momento pensás cuántas personas hay que hacen lo mismo. Y te enojas porque vos ponés esfuerzo en hacer, aunque sea una idiotez como tirar los papeles en un cesto, y te das cuenta que tu esfuerzo no vale".

b. Mesías: los estudiantes esperan que surja una persona que pueda controlar el desorden social e imponga un buen ejemplo ante el malestar social y así generar un cambio masivo.

Ejemplos: (a) "yo creo que tenés que tener un modelo firme, muy fuerte para cambiar todo. Tiene que ser alguien que realmente se pueda imponer y demostrarles a todos que se puede cambiar, que diga 'miren, se puede cambiar y miren todo lo que puede pasar"; y (b) "yo creo que es muy difícil que una sociedad, que un país, esté de acuerdo con algo, para establecerlo tiene que haber alguien que se imponga y que haga las cosas bien, porque es muy difícil que todos estén de acuerdo".

c. Espacio para la discusión de las normas: los participantes creen que se deben generar espacios para que las personas puedan opinar, discutir y plantear alternativas al funcionamiento normativo. De esta manera, las normas ya no tendrían un cariz negativo ni autoritario pues contemplarían a la mayoría de las personas y se generarían de manera democrática.

Ejemplos: (a) "yo creo que no hay espacios y por eso creo que la norma es tan arbitraria, surge en vista de un problema social y bueno, (...) como que no hay espacios en la sociedad institucionales si se quiere decir, no existe. Uno puede 'charlar', los chicos en el secundario pueden 'charlar' en grupo, en la escuela, pero espacios no veo"; (b) "impotencia porque por ahí, no está tanto la chance de participar activamente. No está ese lugar para participar en el consenso sino para denunciar el desacuerdo con la norma y por ahí, cumplimos la norma porque mis valores son que está la norma y por eso hay que cumplirla, supongamos, pero si no estoy de acuerdo quiero tener un lugar en el que pueda expresarlo y hacerlo, y no siento que están esos lugares, yo no lo siento en general, y por ahí lo veo más expresado en cosas masivas que terminan en lugares más agresivos como protestas y ya no hay otra manera de encararlo".

d. Educación: los participantes manifestaron que el cambio devendría mediante la educación. Esto posibilitaría la comprensión de las normas $y$, en segunda instancia, que sean aceptadas voluntariamente y no por imposición.

Ejemplos: (a) "(...) no sé cuál es el alcance ni la manera pero la variable que nos permitiría estar en mejores condiciones y construir nuestras propias normas, o por lo menos controlar la gestión de aquellos que las construyen y nos representan, tiene que ver con la educación., o sea con un nivel educativo importante. (...) pero hay un básico que no sé cuál es el alcance y no lo podría cuantificar pero me parece que lo único que puede contribuir a tener una sociedad que puede consensuar sus normas, a acatar esas normas por propia voluntad, y no porque haya otro que vaya a castigarte, es la educación"; y (b) "hay una cuestión de que tendemos a pensar o a sentir que la norma no es para uno, como que está impuesto un poco desde arriba y arbitrariamente, quizás sea así, sobretodo sea así cuando se la tiene que imponer porque no se la cumple, digamos, la norma lo que sería bueno, no se, pensar un poco que es para uno, esa sería la forma en la que la educación tendría que accionar. Si uno crece pensando que no pararse en la calle es bueno para vos, y no que es algo que hacés para cumplir, eso sería un modo, como decías vos, de interiorizar la norma".

\section{Discusión}

A partir de los resultados expuestos, se observa que los participantes definen a las normas como ejes directrices que ordenan el funcionamiento de un sistema. Sin embargo, cuando circunscriben su función al ámbito argentino, éstas adoptan características negativas, casi contrapuestas a la mencionada anteriormente, y hacen referencia a la falta de sentido (ridículas, inventadas, negativas) o a un sentido restringido (dependientes del contexto o para pocos). Los 
estudiantes creen que las normas no están hechas para cumplimentar sus necesidades o que en su conjunto, no guardan coherencia unas con otras, generando un sistema normativo carente de fundamento.

La característica negativa de la norma está condicionada por la estructura normativa de las instituciones. Según los participantes, el sistema normativo argentino y sus instituciones, se rigen por un doble funcionamiento: uno formal y otro informal. Por lo cual, los ciudadanos deben comprender no sólo las normas por las cuales deberían regirse, y ser sancionados, sino comprender las normas que gobiernan el sistema. Se complejiza la situación porque entre ellas no guardan coherencia. Esto no sólo genera incertidumbre sino una necesidad de que las personas aprendan a manejarse a partir de la socialización de las normas tácitas de las instituciones, lo que conlleva un proceso arduo o la necesidad de otros para comprender el sistema. Tomando los aportes de Cialdini et al. (1990), las personas visualizan que las normas descriptivas no está en concordancia con las normas prescriptivas de la institución.

Relacionando con la propuesta del modelo de norma perversa de Fernández Dols (1992), se crean normas que son incumplibles en sí mismas y se mantienen en vigencia a pesar de su condición. En este sentido, el sistema sostiene prácticas informales que desacreditan las normas institucionales y acrecientan el poder coyuntural de quien gestiona. Esto perjudica al sistema en general, y en particular a las normas que sí funcionan. Como dirían Lauth (2000) y Helmke y Levitsky (2004) se visualiza un funcionamiento ineficaz de las instituciones formales y las instituciones informales son quienes organizan el comportamiento colectivo.

En correspondencia a este problema, los participantes plantearon la generalidad de la trasgresión normativa. Según ellos, las normas se transgreden constantemente y de manera generalizada en todos los ámbitos de la sociedad. Estos datos están en concordancia con las encuestas a nivel nacional del Latinobarómetro (1996-2010); y se relacionan con estudios locales previos que indican una disfuncionalidad institucional y una integración deficitaria del sistema social (Beramendi et al., 2012; Muratori et al., 2013).

La creencia de la transgresión como una práctica generalizada funciona como una manera de justificar el propio incumplimiento y ayuda a tolerar el incumplimiento ajeno. Sin embargo, la sumatoria de las diversas razones del incum- plimiento (i.e., la corrupción, la falta de ejemplo por parte de la autoridad en el cumplimiento de la norma, la injusticia del sistema legal, la falta de recompensa y castigo para quien hace bien las cosas y para quien no lo hace, entre otras) configura un sistema inmutable e impermeable a los cambios. Por esta razón, los participantes no visualizan que pueden confrontar mediante los caminos formales si una institución o una persona incumplen lo pautado, sino que actúan transgrediendo otra norma que los beneficia a ellos pero que no resuelve el problema principal. Esta forma de comportarse se asemeja a lo que Wright (2001) denominó acciones no normativas individuales. Estas acciones se realizan cuando la gente cree que el sistema es injusto, que las desigualdades sociales son profundas, y que el sistema no es permeable al cambio.

Los participantes de los grupos focales opinan que es difícil vislumbrar el cambio social por varias razones: (a) se genera un círculo vicioso donde las personas no pueden marcar cambios con sus conductas debido a una estructura social que los condiciona; (b) apelan al surgimiento de una persona que salve al país mediante la imposición de un modelo; (c) creen en formatos de discusión que se relacionarían más con un modelo democrático directo, que con uno representativo en el cual las personas se comprometen menos política y socialmente (Bobbio, 2004), y concretamente no visualizan que sea viable; $y$ (d) piensan que la educación podría cambiar el sistema social, ya que implicaría un reordenamiento general de éste pero lo manifiestan de manera abstracta, casi utópica. Los estudiantes no perciben que los cambios sociales puedan devenir de un proceso de cambio colectivo sino individual.

Lo que se desprende de los diversos puntos y que remarcaron los estudiantes de los grupos focales, es la desconfianza en el funcionamiento del sistema normativo pues se maneja mediante políticas corruptas, a través de instituciones ineficaces, y con normas contradictorias; mayoritariamente creen que el sistema está pensado para la conveniencia de unos pocos, de los políticos y de ciudadanos con poder, y que excluye las realidades de la mayoría de los argentinos. Básicamente, caracterizan al sistema como injusto.

A partir de estos hallazgos, se infiere que hay un debilitamiento de la legitimidad de las instituciones y de las autoridades (Murphy \& Tyler, 2008; Tyler, 2001, 2006) pues la percepción de corrupción (Rose-Ackerman, 2001; Seligson, 2002), la baja confianza institucional 
(Baker, 2008; González de la Vega et al., 2010) y la percepción de que el proceso de regulación institucional no es justo (Napier \& Tyler, 2008) sostienen dicha creencia.

Esto promueve que los participantes de este estudio perciban que las autoridades, las normas y el sistema en general son autoritarias y que las personas construyan sus propias normas bajo una moral individual donde las normas prescriptivas personas pasan a cobrar un rol primordial. Como plantean Sacconi y Faillo (2010) en los contextos donde la transgresión es alta, la motivación endógena para el cumplimiento normativo pasa a ser un factor relevante. Para los participantes el respeto de la norma es válido cuando concuerdan con sus valores o creencias, cuando les conviene, o cuando depende de la evaluación que hacen a partir del contexto y de la situación. Este relativismo se ve reforzado por la falta de control y de sanción institucional o social, pues creen que sus actos, salvo que estén afectando a alguien en concreto, no perjudican al sistema sino que es una forma de actuar, que no debería ser así, pero es la manera en la cual funciona el sistema actual.

Los estudiantes dan respuestas cínicas, que remarcan sentimientos de desmoralización, en relación a cómo se debe enfrentar este escenario, asumiendo la gran mayoría que el cambio devendrá a partir de cambios estructurales o sociales, pero prevalece, sin embargo la desconfianza de que ellos sucedan. Al mismo tiempo, aseguran que la forma de conducirse en el sistema institucional argentino es mediante un camino individual, con un sistema propio de normas que contempla la transgresión y que, al mismo tiempo procura no perjudicar a alguien concretamente.

De esta manera, para abordar la temática normativa no alcanza con el estudio de las actitudes individuales hacia las normas, las autoridades y las instituciones, pues los efectos de la legitimidad en relación a la estabilidad social es la resultante de un proceso colectivo que involucra a los otros. Los resultados dan cuenta de una norma situada en una cultura, en la que la transgresión es una pauta de comportamiento socialmente aceptada, producto de una determinada percepción del sistema normativo. En este marco, se abre una oportunidad para replantear cuáles son las creencias sociales compartidas sobre el sistema argentino y cómo esto repercute en la calidad de vida y bienestar de cada ciudadano.

\section{Referencias}

Abello, R., Amarís, M., Blanco, A., Madariaga, C., Díaz, D. \& Arciniégas, T. (2008). Bienestar, Autoestima, Depresión y Anomia en personas que no han sido víctimas de violencia política y social. Investigación y Desarrollo, 16(2), 214-231.

Aceituno, R., Asún, R., Ruiz, S., Reinoso, A, Venegas, J. I. \& Corbalán, F. (2009). Anomia y Alienación en Estudiantes Secundarios de Santiago de Chile: Resultados Iniciales de un Estudio Comparativo 1989-2007. Psykhe, 16(2), 3-18. doi:10.4067/s0718-22282009000200001

Aguinis, M. (2001). El atroz encanto de ser argentino. Buenos aires: Planeta.

Alonso, D. R., \& Brussino, S. A. (2012). Legitimidad y percepciones generales de justicia de las autoridades legales de Córdoba (Argentina): Un abordaje desde las valoraciones de justicia procedimental. Interdisciplinaria, 29(2), 271-286.

Baker, A. (agosto, 2008). State Government Performance and Citizen Trust. The annual meeting of the APSA 2008, Hynes Convention Center, Boston, Massachusetts.

Beramendi, M. (2014). Percepción del sistema normativo, transgresión y sus correlatos psicosociales en Argentina (Tesis doctoral inédita). Facultad de Psicología. Universidad de Buenos Aires, Ciudad Autónoma de Buenos Aires.

Beramendi, M., Sosa, F., \& Zubieta, E. (2012). Anomia y percepción de control en el contexto argentino. Revista Psicología Política, 45(2), 43-60.

Beramendi, M., \& Zubieta, E. (2015). A proposal to analize social transgresión and its psychosocial consequences. Manuscrito enviado para su publicación.

Blank, T. (2003) Determinants of National Identity in East and West Germany: An Empirical Comparison of Theories on the Significance of authoritarianism Anomie, and General Self-Esteem. Political Psychology, 24(2), 259-288. doi:10.1111/0162-895x.00328.

Bobbio, N. (2004). El futuro de la democracia. México: Fondo de la Cultura.

Brandt, M. (2013). Do the Disadvantaged Legitimize the Social System? A Large-Scale Test of the Status-Legitimacy Hypothesis. Journal of Personality and Social Psychology, 104(5), 765-785.

Centro de Opinión Pública de la Universidad de Belgrano (COPUB, 2013). Qué piensa la gente de la Corrupción. Recuperado de http://new.pensamientopenal.com.ar/ sites/default/files/2013/06/003_copub_-_documento_-_que_piensa_la_gente_sobre_la_corrupcion.pdf.

Cialdini, R. B., Reno, R. R., \& Kallegren, C. A. (1990). A focus theory of normative conduct: Recycling the concept of norms to reduce littering in public places. Journal of Personality and Social Psychology, 58, 1015-1026. doi: 10.1037/0022-3514.58.6.1015

Cialdini, R.B. (2007). Descriptive social norms as underappreciated sources of social control. Psychometrika, 72(2), 263-268. doi: 10.1007/s11336-006-1560-6

De Biase, T. (3 de abril de 2010). "Sentimos que no hay ley ni autoridad". La Nación. Recuperado de http://www. lanacion.com.ar/1250156-sentimos-que-no-hay-ley-niautoridad.

Denevi, M. (2002). La viveza, entre la inteligencia y la estupidez. Boletín del Centro Naval, 120(804), 31-32.

Durkheim, E. (1998). El Suicidio. Buenos Aires: Grupo Editorial Tomo.

Edelman (2013). Índice de Confianza Edelman. Recuperado de http://www.edelman.com/insights/intellectual-property/ trust-2013/trust-around-the-world/.

Fernández Dols, J. M. (1992). Procesos escabrosos en Psicología Social: el concepto de norma perversa. Revista de Psicología Social, 7(2), 243-255. 
Fernández Dols, J. (1993). Norma perversa: hipótesis teóricas. Psicothema, 5(Supl. 1), 91-101.

González de la Vega, A., Quintanilla, A. \& Tajonar, M. (2010). Confianza en las instituciones políticas mexicanas: ¿capital social, valores culturales o desempeño? En A. Moreno (Ed.), La confianza en las instituciones. México en perspectiva comparada (pp 63-99). Ciudad de México: Centro de Estudios Sociales y de Opinión Pública.

Helmke, G., \& Levitsky, S. (2004). Informal Institutions and Comparative Politics: A Research Agenda. Perspectives on Politics, 2(4), 725-740. doi:10.4337/9781781001219.00011

Herrera, C. B. (31 de diciembre de 2004). La falta de credibilidad en la Justicia. El independiente. Recuperado de http://www.elindependiente.com.ar/papel/hoy/archivo_2004/noticias_v.asp?32335.

Jauretche, A. (1973). Manual de las Zonzeras Argentinas. Buenos Aires: a.peña lillo editor s.r.l

Laca Arocena, F., Mejía Ceballos, J. C., \& Yañez Velasco, C. (2010). Identidad mexicana e interés político: Predictores de bienestar social y anomia. Acta Universitaria, 20(2), 40-49.

Latinobarómetro (1996-2010). Análisis en Línea: Igualdad ante la ley - derechos y obligaciones. Recuperado de http:// www.latinobarometro.org/latino/LATAnalizelndex.jsp.

Lauth, H-J. (2000). Informal institutions and democracy. Democratization, $7(4), 21-50$. doi:10.1080/13510340008403683

MacCoun, R. J. (1993). Drugs and the Law: A Psychological Analysis of Drug Prohibition. Psychological Bulletin, 113(3), 497-512. doi:10.1037/0033-2909.113.3.497.

Mafud, J. (1965). Psicología de la viveza criolla. Buenos Aires: Américalee.

Manstead, A. S. R. (2000). The role of moral norm in the attitudebehavior relation. En D. J. Terry \& M. A. Hogg (Eds.), Attitude, behavior, and social context: The role of norms and group membership (pp. 11-30). Mahwah, NJ: Erlbaum.

Monsegur, S., Espinosa, A., \& Beramendi, M. (2014). Identidad nacional y su relación con la Dominancia Social y la Tolerancia a la Transgresión en Buenos Aires. INTERDISCIPLINARIA, 31(1), 5-23. doi:10.16888/interd.2014.31.1.1

Mueller, C. ,\& Landsman, M. (2004). Legitimacy and Justice Perceptions. Social Psychology Quarterly, 67(2), 189-202. doi:10.1177/019027250406700205.

Muñoz Justicia, J. (2005). Análisis cualitativo de datos textuales con ATLAS TI. 5. Barcelona: Universidad Autónoma de Barcelona.

Muratori, M., Delfino, G. I., \& Zubieta, E. (2013). Percepción de anomia, confianza y bienestar: la mirada desde la psicología social. Revista de Psicología, 31(1), 129-150.

Murphy, K., \& Tyler, T. (2008). Procedural justice and compliance behavior: the mediating role of emotions. European Journal of Social Psychology, 38, 652-668. doi:10.1002/ejsp.502.

Napier, J., \& Tyler, T. (2008). Does Moral Conviction Really Override Concerns About Procedural Justice? A Reexamination ode the Value Protection Model. Social Justice Research, 21, 509-528. doi:10.1007/s11211-008-0083-y

Nino, C. (2005). Un país al margen de la ley. Buenos Aires: Ariel.

Observatorio de la Deuda Social Argentina $(2012,2013)$. Barómetro de la Deuda Social Argentina: Serie del Bicentenario (2010-2016).
O'Donnell, G. (1996). Illusions About Consolidation. Journal of Democracy, 7(2), 34-51. doi:10.1353/jod.1996.0034.

Onwuegbuzie, A. J., Dickinson, W. B., Leech, N. L., \& Zoran, A. G. (2011). Un marco cualitativo para la recolección y análisis de datos en la investigación basada en grupos focales. Paradigmas, 3, 127-157.

Rose-Ackerman, S. (2001). La corrupción y los gobiernos: Causas, consecuencias y reforma. Madrid: Siglo XXI.

Roselli, N. (2000). Representación social de "los argentinos", "los españoles", "los latinoamericanos" y "los europeos" en estudiantes universitarios argentinos. Revista Latinoamericana de Psicología, 32(1), 127-160.

Sacconi, L., \& Faillo, M. (2010). Conformity, reciprocity and the sense of justice. How social contract-based preferences and beliefs explain norm compliance: the experimental evidence. Const Polit Econ, 21, 171-201. doi:10.2139/ ssrn.1163742

Srole, L. (1956). Social integration and certain corollaries: an exploratory study. American Sociological Review, 21, 709-716. doi:10.2307/2088422.

Strauss, A., \& Corbin, J. (2002). Bases de la investigación cualitativa. Técnicas y procedimientos para desarrollar la teoría fundamentada. Colombia: Editorial Universidad de Antioquía.

Tyler, T. (2001). Public trust and confidence in legal authorities: What do majority and minority group members want from the law and legal authorities? Behavioral Science \& the Law, 45, 5-24. doi:10.1002/bsl.438.

Tyler, T. (2006). Psychological Perspectives on Legitimacy and Legitimation. Annual Review of Pshychology, 53, 375-400. doi:10.1146/annurev.psych.57.102904.190038

United Nations Organization for Education, Science and Culture [UNESCO], (2010). Datos mundiales de educación VII Ed. 2010/11. Recuperado de http://www.ibe.unesco. org/fileadmin/user_upload/Publications/WDE/2010/pdfversions/Uruguay. $\overline{p d f}$

White, K., Smith, J.,Terry, D., Greenslade, J., \& McKimmie, B. (2009). Social influence in the theory of planned behaviour: The role of descriptive, injunctive, and in-group norms. British Journal of Social Psychology, 48, 135-158. doi:10.1348/014466608×295207

Wolfe, R. (1968). Two views of anomia and the nature of normlessness. The Journal of Social Psychology, 75, 91-95. doi:10.1080/00224545.1968.9712477

Wright, S. \& Taylor, D. (1998). Responding to Tokenism: individual action in the face of collective injustice. European Journal of Social Psychology, 28, 647-667. doi:10.1037/0022-3514.58.6.994

Wright, S. (2001). Tokenism, Ambuity and the Tolerance of Injustice. En J. Jost \& B. Major (Eds.), The Psychology of Legitimacy (pp. 223-254). Estados Unidos: Cambridge University Press.

Wright, S., Taylor, D. y Moghaddam, F. (1990). Responding to membership in a Disadvantaged Group: From Acceptance to Collective Protest. Journal of Personality and Social Psychology, 58(6), 994-1003. doi:10.1037/00223514.58.6.994

Para citar este artículo:

Beramendi, M., \& Zubieta, E. (2015). Un estudio exploratorio sobre la relación entre la legitimidad institucional y la trasngresión normativa en Argentina. Ciencias Psicológicas 9(1): 15 - 26 